\title{
Table ronde : vers une vision européenne des politiques de solidarité
}

\section{Nicole Denoit}

\section{(2) OpenEdition}

1 Journals

Édition électronique

URL : http://journals.openedition.org/communicationorganisation/2381

DOI : 10.4000/communicationorganisation.2381

ISSN : $1775-3546$

Éditeur

Presses universitaires de Bordeaux

Édition imprimée

Date de publication : 1 mai 2000

ISSN : 1168-5549

Référence électronique

Nicole Denoit, «Table ronde : vers une vision européenne des politiques de solidarité », Communication et organisation [En ligne], 17 | 2000, mis en ligne le 27 mars 2012, consulté le 20 avril 2019. URL

http://journals.openedition.org/communicationorganisation/2381 ; DOI : 10.4000/

communicationorganisation.2381

Ce document a été généré automatiquement le 20 avril 2019.

(c) Presses universitaires de Bordeaux 


\title{
Table ronde : vers une vision européenne des politiques de solidarité
}

\author{
Nicole Denoit
}

\section{NOTE DE L'ÉDITEUR}

Compte rendu deNicole Denoit

1 Les 4 et 5 février 1999, l'Institut du Mécénat de Solidarité organisait, au CNIT à Paris, les Rencontres Humagora 99 sur le thème « compétitivité économique et cohésion sociale, un nouvel enjeu pour l'entreprise ». Les quelque deux mille visiteurs qui participèrent à ces rencontres, purent assister à une table ronde dont Nicole Denoit nous a rapporté le compte rendu.

2 La table ronde était animée par Gabriel Milesi, responsable du Journal de l'Économie d'Europe 1 et rassemblait :

3 Peter Davies, Managing Director of BITC (Business In The Community)

4 Étienne Davignon, Président de la Société Générale de Belgique et de l'EBNSC (European Business Network for Social Cohésion)

5 Jean-Baptiste de Foucauld, Président de SNC (Solidarités Nouvelles face au Chômage)

6 Jean-Marc Genis, Président de Marks \& Spencer France

7 David Halley, Consultant de BITC

8 Jean-Paul Tricart, Commission européenne, DG V

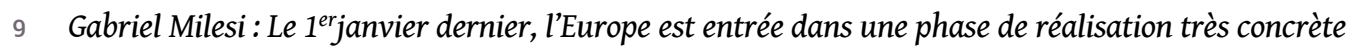
avec l'avènement de l'euro. Nous sommes réunis aujourd'hui pour évoquer de quelle manière cette convergence européenne peut se traduire en matière de mécénat humanitaire. 
10 Jean-Baptiste de Foucauld: Le succès de l'euro ne suffira pas. Le fait est que les pays européens, et tout particulièrement la France, peinent à (re)trouver un modèle socioéconomique performant. Nos sociétés sont prises dans un double mouvement de globalisation et d'atomisation. Trois camps s'opposent : celui de l'immobilisme, celui de la dérégulation et enfin celui qui plaide pour de nouvelles régulations sociales et institutionnelles.

11 Tous les acteurs ont un rôle à jouer pour sortir de cette situation: aussi bien les entreprises que l'État, les associations ou les citoyens. C'est sur ce principe de citoyenneté active qu'a été fondée "Solidarités Nouvelles face au Chômage " (SNC) qui aide des chômeurs à retrouver le chemin de l'emploi par un accompagnement amical et pour un partage de revenus créateur d'emploi dans le secteur associatif. Les entreprises, elles, doivent contribuer à définir les nouvelles régulations.

12 En 1992, SNC avait fait signer et diffuser un texte sur l'implication nécessaire de l'entreprise dans la lutte contre l'exclusion sociale en termes, par exemple, de recrutement, de gestion des licenciements ou de développement local. L'initiative avait concerné d'importants chefs d'entreprise comme Jean Gandois ou Francis Mer. Mais elle était restée ponctuelle et n'avait pas concerné le patronat dans son ensemble. Aujourd'hui encore, la position du Medef sur ce sujet n'est pas claire. Le "social» continue à être considéré comme un sous-produit de l'« économique ».

13 C'est dans ce contexte qu'a été lancée sous l'impulsion de Jacques Delors l'heureuse initiative du Réseau européen des entreprises pour la cohésion sociale.

14 Nous devons désormais nous demander comment aller plus loin, en tenant compte de certaines évolutions récentes.

15 - Les entreprises françaises, à l'inverse de leurs homologues du Royaume-Uni, de l'Europe du Nord, et à l'exception notable de France Télécom, tendent à ne plus être assez présentes dans le réseau.

16 - Le développement du réseau lui-même doit être approfondi. Étienne Davignon, son président, $\mathrm{y}$ reviendra sans doute.

17 - Il conviendrait d'étudier tout particulièrement les relations entre les entreprises et leurs actionnaires, au premier rang desquels nous entendons faire figurer les fonds de pension. Leurs analyses souvent frustes et la tendance au mimétisme décisionnel ont des conséquences dévastatrices pour l'emploi.

18 Aujourd'hui, le manager l'emporte sur le créateur de valeurs. Le monde de la finance est plus que jamais déconnecté du monde des hommes. Ce thème est l'objet d'un cahier spécial de la revue «Échanges et Projets » financé par l'EBNSC et d'un manifeste que nous avons fait circuler au début de 1997. Nous voulons susciter la constitution, au sein de l'EBNSC, d'un réseau de financiers décidés à se préoccuper des relations entre la sphère financière et la sphère " réelle ».

19 Il faut également envisager la création d'indicateurs mesurant le niveau et la qualité de l'action sociale des entreprises. Ces indicateurs pourraient figurer en sus des traditionnels ratios financiers. Aux États-Unis, il est devenu assez courant de faire figurer dans les bilans des entreprises des indicateurs concernant le développement durable. Nous suggérons la mise sur pied d'un prix récompensant la meilleure initiative en la matière. 
20 La réussite de l'euro est à l'origine d'un nouvel élan en termes de croissance économique. Elle doit aussi être l'occasion d'un nouvel élan social, car l'avènement de la monnaie unique est un exemple éclatant du succès de la volonté politique, qui démontre ainsi sa capacité à dépasser les contraintes économiques.

21 C'est pourquoi, nous avons élaboré un nouveau manifeste sur ce sujet qui a reçu l'appui de Jacques Santer. Il a circulé avec un certain succès en France mais son écho au niveau européen demeure faible.

22 Étienne Davignon: A l'origine de l'EBNSC se trouve la déclaration sur la responsabilité sociale de l'entreprise, rédigée sous l'égide de Jacques Delors et diffusée ensuite avec succès dans les milieux économiques de la Communauté Européenne, au début de 1995. Dans un deuxième temps, nous avons cherché à mesurer les dispositions réelles des entreprises en la matière. Nous avons pour cela réalisé une étude, avec l'aide de la Commission Européenne, qui a mis en lumière plusieurs points: tout d'abord, les responsables d'entreprises européens considèrent qu'il est normal d'associer la performance économique et la responsabilité sociale. En Grande-Bretagne notamment, le corporate citizenship est une réalité concrète depuis un certain temps déjà. Par ailleurs, ces mêmes responsables souhaitent éviter le système de la "cotisation de la bonne conscience ».

23 À la suite de ces initiatives, le Réseau européen pour la cohésion sociale (EBNSC) a été lancé officiellement en octobre 1996 par le président de la Commission Européenne Jacques Santer. Il s'articule principalement autour d'un centre européen de ressources sur le monde des affaires et la cohésion sociale. Il s'agit d'une banque de données, accessible par Internet à l'adresse www.ebnsc.org, rassemblant notamment les descriptifs des opérations menées par les entreprises membres dans le cadre de l'exercice de leur «responsabilité sociale» (promotion de l'intégration sur le marché du travail, amélioration de la formation professionnelle, lutte contre l'exclusion, réduction des licenciements, développement de nouveaux métiers, etc.).

24 L'EBNSC est un réseau pleinement opérationnel. L'une de ses originalités est qu'il relie des praticiens d'entreprise qui s'efforcent de mettre en commun leurs expériences afin de ne pas avoir à «réinventer la roue » à chaque opération. L'accent est également mis sur l'association avec de grandes universités de gestion.

25 Il faut noter que le réseau européen se présente en pratique comme une fédération de réseaux nationaux. En effet, nous souhaitons ne pas encourir le reproche d'une uniformisation «bruxelloise » des projets. Nous accordons le plus grand soin à obtenir l'accord des acteurs concernés sur le message à transmettre à propos de chaque projet présent dans la banque de données EBNSC. Pour cela, nous entendons faire preuve d'une pédagogie optimale.

26 Afin de développer le réseau, nous souhaitons promouvoir le dialogue entre les entreprises et les pouvoirs publics, notamment dans le but de lever certains obstacles administratifs. Le président Jacques Santer partage ce souhait puisqu'il a demandé à la fin de l'année dernière l'organisation d'une conférence réunissant entreprises et pouvoirs publics européens.

Il importe de ne pas oublier que le succès de l'EBNSC ne se mesure pas au seul nombre des entreprises affiliées au réseau mais à celui des entreprises réellement actives en matière de cohésion sociale. 
Jean-Paul Tricard: Le maintien nécessaire d'un lien entre la performance économique et la cohésion sociale est un des plus importants fils conducteurs de la réflexion de la Commission. Les solutions ne sont pas aisées à trouver mais elles existent.

En outre, le traité d'Amsterdam met de nouveaux moyens à la disposition des instances européennes en matière de politique de l'emploi et de lutte contre l'exclusion. Le nouveau traité devrait plus que jamais permettre de mettre sur pied une véritable stratégie européenne pour l'emploi. Un chapitre spécifique du traité d'Amsterdam est consacré à ce sujet. Il comporte quatre "piliers", c'est-à-dire quatre objectifs fondamentaux : la promotion de «l'employabilité»; de l'adaptabilité ; de la culture d'entreprise ou « entreprenariat »; enfin de l'égalité des chances entre hommes et femmes en matière d'accès au monde du travail.

o Jamais le domaine de l'emploi n'avait été reconnu à ce point comme une question d'intérêt commun. Nous allons pouvoir travailler à la mise en place d'une action concertée sur ce sujet. Nous souhaitons encourager l'auto-évaluation par les pays membres des processus qui seront mis en place. Nous souhaitons également promouvoir l'association des partenaires sociaux et des entreprises. D'une manière générale, nous souhaitons aller au-delà des positions de principe souvent généreuses, mais insuffisamment traduites dans les faits.

1 La Commission Européenne s'oriente vers la mise en place d'un Pacte pour l'Emploi. C'est d'ailleurs l'existence d'une réunion importante consacrée à ce projet qui explique l'absence aujourd'hui à cette tribune de la directrice générale adjointe de la DG V, Odile Quintin.

Ainsi que l'évoquait Monsieur Davignon, la Commission projette également l'organisation d'une journée de travail en 1999 pour promouvoir les échanges entre entreprises et gouvernements.

3 Le président Santer souhaite en outre s'appuyer précisément sur le rapport «Gilenhamar », consacré à l'impact de la fermeture de l'usine de Renault Vilvoorde pour promouvoir un mode de gestion plus «socialement responsable» des mutations industrielles : il s'agit d'encourager une anticipation des changements et leur préparation par le biais d'un dialogue renforcé et d'une meilleure information des personnels concernés.

34 Toutes les mesures visant à la promotion du corporate citizenship qu'évoquait Étienne Davignon, par exemple à travers l'instauration de la publication annuelle d'un rapport social (ou sociétal) par les entreprises, sont également considérées ave beaucoup d'intérêt par la Commission européenne.

5 En conclusion, je souhaite revenir sur l'idée que le traité d'Amsterdam marque l'amorce d'un tournant positif. La Commission fut très présente sur le terrain de la lutte contre l'exclusion dans les années 1980. La situation connut ensuite un blocage regrettable. Le nouveau Traité doit être l'occasion d'une relance de cette politique. Un programme précis sera d'ailleurs annoncé en juillet ou septembre de cette année.

L'article 13 du Traité, consacré à la lutte contre les discriminations pour des raisons ayant trait aux handicaps, à la religion, à l'origine ethnique ou à l'orientation sexuelle d'une personne devrait notamment engendrer la proposition par la Commission de directives précises et d'un programme d'actions concrètes. 

expérience que nous souhaitons partager et échanger avec nos partenaires européens. C'est le sens de notre présence au sein de l'European Business Network for Social Cohésion (EBNSC). Nous avons également mis en place un programme de séminaires de présentation et de formation qui se déroulent dans les ambassades britanniques en Europe. L'étape parisienne qui au lieu dernièrement a été co-organisée avec beaucoup de succès par l'TMH. Le prochain séminaire aura lieu à Madrid au printemps prochain. 


\section{d'un siècle, l'idée selon laquelle les métiers de la grande distribution sont par nature} tournés vers le consommateur et donc l'implication dans la cité.

\section{L'historique}

Michael Marks, exilé polonais, fondateur de la société en 1890, a dès l'origine fait de l'humanitaire l'un des axes principaux de la culture de son entreprise. Un système de restaurants subventionnés et d'assistance médicale aux collaborateurs ainsi qu'à leurs familles a été mis en place dès les années 1920. En matière sanitaire, les efforts portent actuellement avant tout sur le dépistage des cancers et la lutte contre le tabagisme.

51 Fait plus significatif encore, ces avancées sociales ont été imposées aux partenaires sociaux et aux fournisseurs de l'entreprise. C'est ainsi que l'exemple de Marks \& Spencer a pu faire «tache d'huile » tout au long de ce siècle, et ce pratiquement à l'échelle de la Grande-Bretagne dans son ensemble.

\section{La situation actuelle}

52 En 1998, 100 millions de francs ont été consacrés par l'entreprise à l'action humanitaire. Dans les années 80, les priorités concernaient la vie dans les cités, les jeunes, l'emploi. Aujourd'hui, l'entreprise entend se préoccuper essentiellement de protection de l'environnement, de santé, d'aide aux sans-abri et de lutte contre la toxicomanie.

53 Nous considérons que la mise en place d'un accompagnement est indissociable du don d'argent. C'est pourquoi nous avons opté pour une politique de détachement de notre personnel. Nos collaborateurs, habitués à considérer ces périodes de détachement comme appartenant au déroulement normal d'une carrière, ont ainsi l'occasion de vivre une expérience très enrichissante de découverte du monde associatif. Au retour des personnes concernées, cet enrichissement personnel profite bien évidemment à l'entreprise.

54 Par ailleurs, notre capacité de financement et de détachement étant limitée, nous ne prétendons pas pouvoir nous passer des fonds publics. Actuellement, 35 personnes à temps plein et 200 à temps partiel sont détachées et travaillent sur les projets que nous avons sélectionnés. Cela correspond à un engagement financier de 23 millions de francs.

L'une de nos directrices des ressources humaines est ainsi mise à la disposition de l'IMH. Un autre de nos directeurs, qui a par le passé collaboré aux Restaurants du Cœur, travaille depuis le 31 décembre dernier à la Mission locale pour l'emploi de Poissy. En matière de temps partiel, nous

\section{Remarques conclusives}

56 Notre entreprise est présente dans 12 pays, en majorité nord européens (Belgique, Allemagne) mais aussi méridionaux (Espagne). La France apparaît comme dotée d'une culture plutôt singulière : en matière d'action humanitaire, il est très souvent et très naturellement fait en priorité appel à l'État. Néanmoins, Marks \& Spencer y applique les mêmes principes qu'en Grande-Bretagne, et ce avec succès. 
57 Cela dit, je constate que notre tâche consiste également à faire face au scepticisme ambiant, qui s'est encore manifesté dernièrement par la publication dans un grand quotidien français que je ne citerai pas d'un article pour le moins acerbe.

Gabriel Milesi : En m'abstenant également de citer le nom du journal concerné, j'indique le titre de l'article auquel vous venez de faire allusion. Il s'agit de : «Humagora 99 : le capital fait sa B.A ».

J'en profite pour vous inviter à approfondir la question: est-ce bien aux entreprises qu'il revient d'agir dans le domaine de l'action sociale et humanitaire?

60 Jean-Marc Genis: A mon sens, aucune action politique ne peut remplacer l'initiative individuelle, privée. Bien sûr, il existe un intérêt direct pour les entreprises à agir dans le domaine humanitaire: M. Davies a cité la phrase du avons mis en place le programme « 100 heures pour seconder » : nous accordons 100 heures, sur leur temps de travail, à ceux de nos agents de maîtrise qui veulent s'investir dans une association. Parmi les exemples récents, je citerai CANA78, SOS Accueil et la Mission locale de Versailles.

61 Une autre de nos modalités d'action consiste à encourager la collecte de fonds. Marks \& Spencer double, jusqu'à concurrence de 150000 francs, les sommes recueillies par nos collaborateurs au cours des manifestations qu'ils organisent, des lotos ou des matchs de gala par exemple.

62 Marks \& Spencer reçoit environ 400 demandes d'aide par semaine. Celles-ci sont traitées par un comité de sélection dans lequel sont représentés tous les niveaux hiérarchiques de l'entreprise fondateur de Marks \& Spencer sur les liens existant entre une "société saine » et un « commerce sain ». Par ailleurs, l'intérêt de l'immersion des collaborateurs dans le monde associatif est indéniable. Mais cela ne justifie évidemment pas qu'on nous fasse ces mauvais procès.

63 Étienne Davignon: Ces commentaires sur le cynisme supposé des entreprises actives dans le domaine humanitaire m'exaspèrent. Ils ressortissent pour moi à cette tout aussi exaspérante propension européenne à commenter sans fin ses échecs ou, en tout cas, les aspects perfectibles de ses actions.

J'ai souvenir également qu'à une époque, l'appartenance à l'EBNSC d'entreprises qui procédaient à des licenciements était stigmatisée. On a pu lire que ces entreprises avaient adhéré par pur souci de se procurer un gage de respectabilité à peu de frais. Ces propos sont sans fondement et bêtement réducteurs. Il est décidément plus que temps de privilégier les actions concrètes sur des discours prétendument intellectuels.

\section{Question de la salle :}

Actuellement, 2 Français sur 10 vivent en dessous du seuil de pauvreté, fixé à 3800 francs de revenus mensuels. L'association dont je suis membre gère 800 comités et 4000 antennes. Nous sommes de véritables professionnels en contact étroit avec le terrain. Pourquoi les entreprises ne s'appuient-elles pas plus directement sur des structures comme les nôtres?

Jean-Baptiste de Foucauld: Le partenariat entre les entreprises et les associations doit incontestablement être développé. Je voudrais par ailleurs revenir sur l'article qu'évoquaient Jean-Marc Genis et Gabriel Milési. Je pense pour ma part qu'il existe effectivement une tradition paternaliste au sein du monde des entreprises, mais elle n'est pas nécessairement condamnable en soi. 
67 La vraie question est à mon sens la suivante : actuellement, nous sommes loin d'un accès universel aux droits sociaux fondamentaux et nous nous en éloignons peut-être de plus en plus ; les entreprises sont-elles réellement conscientes qu'elles doivent participer à la recherche et à la mise en place de nouvelles régulations sociales ? Elles ne devraient pas à mon avis, se contenter d'actions de type humanitaire, mais s'efforcer de bâtir de nouvelles règles du jeu.

La sphère sociale est de plus en plus complexe. Son fonctionnement est désormais fondé sur l'interaction entre des couples notionnels antagonistes: sécurité/flexibilité, initiative/solidarité, etc. Il est essentiel de rendre ceux-ci opérationnels

Gabriel Milesi: M. Davies, comment expliquez-vous ce paradoxe qui veut que coexistent au Royaume-Uni d'un côté l'exemple de votre intense "activisme social » et de l'autre les apparentes réticences de votre gouvernement à harmoniser sa législation dans ce domaine avec les normes européennes?

70 Peter Davies: Les Britanniques sont très conscients du fait que l'État ne peut pas tout gérer. Il existe une responsabilité citoyenne, qui s'exerce au niveau des individus et des entreprises. Ces dernières sont de surcroît assez convaincues par l'idée qu'un certain niveau d'exclusion sociale est incompatible avec une compétitivité économique optimale, et ce pour les raisons de soutien de l'activité que nous avons déjà évoquées, mais aussi parce qu'il existe de véritables gisements de compétences au sein des populations marginalisées dont les entreprises ne sauraient se passer.

\section{Question de la salle :}

71 J'ai cru comprendre que Monsieur de Foucauld prônait la recherche et la mise en place d'un système fixe, rigide par nature, de réponses à la situation de crise sociale que nous traversons. Cette conception est-elle valable aujourd'hui, à l'ère annoncée de la flexibilité ? Ne risque-t-on pas, en raisonnant de cette manière, de se couper de la richesse des initiatives multiformes proposées par un tissu associatif « kaléidoscopique »? Quelle est, de surcroît, la finalité d'un tel discours : ne serait-elle pas d'éviter de remettre en cause une hiérarchie sociale bien établie et dont certains entendent bien ne pas se départir?

72 Jean-Baptiste de Foucauld: Si vous m'avez réellement entendu plaider pour la mise en place d'un modèle uniforme, alors votre critique est amplement justifiée. Cependant, par « modèle ", j'entends « mode de régulation sociale », qui fait toute sa place à la diversité et à l'initiative, mais avec des règles du jeu communes. Jusqu'à la fin de la période dite des «Trente Glorieuses », cette régulation, même si elle était loin d'être parfaite, avait lieu. Aujourd'hui, ce n'est plus le cas: notre Constitution n'est d'ailleurs plus respectée puisque l'accès universel aux droits sociaux fondamentaux (santé, logement, travail...) n'existe plus.

73 Or je pense que le simple jeu du marché ne suffira pas à engendrer de nouveaux modes satisfaisants de régulation sociale. Nous avons donc besoin d'institutions qui puissent réinventer l'articulation entre marché et démocratie. Nous observons deux tendances contradictoires : l'immobilisme, tel qu'il peut être pratiqué actuellement en France, et la dérégulation telle qu'elle existe aux États-Unis, pays prospère mais où la précarité s'accroît. Un « moyen terme » européen reste à trouver. 